\title{
Uric acid lowering effect and pro-inflammatory cytokine modulation along with enhanced renal transporter activities by K-25, a Yokuininto in an endotoxin- induced renal injury condition
}

\author{
Dal-Seok Oh, Seung-Hoon Lee, Gunhyuk Park, Sung Man Oh
}

The K-herb Research Center, Korea Institute of Oriental Medicine, Korea

Background: Prolonged hyperuricemia is associated with kidney disease or gouty arthritis. K-25, a commercially available Yokuininto in Kampo medicine, suppresses obesity in high fat diet-induced obese mice; little is currently known about its known anti-hyperuricemic and anti-inflammatory effects could be applied into the new therapeutic challenge. In this study, we reported that K-25 exerted dual actions on lowering serum uric acid (sUA) levels by blocking pro-inflammatory cytokine activities and inducing uricosuric function with anti-renal injury functions.

Methods: The sUA was measured in potassium oxonate (PO) administered mice. Renal transporter uptake assays were measured by HEK293 cells overexpressing system, MDCKII-BCRP transporter assay and Xenopus oocytes system. Immunoassay was used to detect the molecules (OAT3, GLUT9, XO, NGAL, KIM-1 and IL-1 alpha) in LLC-PK1 cell lines.

Results: K-25 (300 mg/kg) reduced sUA by approximately 44\% compared to that of PO-induced mice. Organic anion transporter 3 (OAT3) levels decreased in PO-induced hyperuricemic condition, whereas glucose transporter 9 (GLUT9) transporter levels were markedly increased. However, the condition did not alter the levels of URAT1. K-25 inhibited the lipopolysaccharide (LPS)-induced secretion of interleukin-1 alpha (IL-1 alpha) by approximately 63.2\% compared to the LPS-treated macrophages. In addition, 500 and $1,000 \mathrm{mcg} / \mathrm{mL} \mathrm{K}-25$ inhibited nitric oxide synthesis by approximately $33.7 \%$ and $64.6 \%$, respectively. K-25 inhibited xanthine oxidase (XO) activity as was already known. Furthermore, 10 $\mathrm{ng} / \mathrm{mL}$ of IL-1 alpha, a pro-inflammatory cytokine, elevated neutrophil gelatinase-associated lipocalin (NGAL) and kidney injury molecule-1 (KIM-1) activities in LLC-PK1 cells. The activities of kidney injury biomarkers, NGAL and KIM -1 , were reduced by K-25 approximately $36.9 \%$ and $72.1 \%$, respectively.

Conclusions: The present findings demonstrated that Yokuininto lowered sUA through both increased uric acid excretion and decreased uric acid production. Our results may provide a basis for the protection of prolonged hyperuricemiaassociated acute kidney injury with traditional uric acid-lowering agents such as Yokuininto. 